\title{
Charge form factors and nucleon shape*
}

\author{
A. J. Buchmann ${ }^{\dagger}$ \\ Institute for Theoretical Physics \\ University of Tübingen \\ Auf der Morgenstelle 14 \\ D-72076 Tübingen, Germany
}

\begin{abstract}
To obtain further information on the geometric shape of the nucleon, the proton charge form factor is decomposed into two terms, which are connected respectively with a spherically symmetric and an intrinsic quadrupole part of the proton's charge density. Quark model relations are employed to derive expressions for both terms. In particular, the proton's intrinsic quadrupole form factor is obtained from a relation between the $N \rightarrow \Delta$ and neutron charge form factors. The proposed decomposition shows that the neutron charge form factor is an observable manifestation of an intrinsic quadrupole form factor of the nucleon. Furthermore, it affords an interpretation of recent electron-nucleon scattering data in terms of a nonspherical distribution of quark-antiquark pairs in the nucleon.
\end{abstract}

PACS numbers: 11.30j, 13.40.Gp, 13.40.Em, 13.60.Rj, 14.20.Gk

\section{ELECTROMAGNETIC PROBING OF NUCLEON STRUCTURE}

Elastic electron-proton scattering experiments done at the Stanford Linear Accelerator about 50 years ago have shown that the proton has a finite size of about $0.9 \mathrm{fm}$. In addition, these and subsequent experiments have provided detailed information on the radial variation of the charge and magnetization densities of the proton [1-4]. Nucleon structure information is encoded in two elastic electromagnetic form factors, which parametrize the deviation of the measured cross section from the theoretical Mott cross section $\sigma_{M}$. The latter describes the scattering of a pointlike spin $1 / 2$ electron on a spinless and structureless nucleon (Fig. 1). In the one-photon exchange approximation, the differential cross section for elastic electron-nucleon scattering, where only electrons scattered into solid angle $d \Omega$ are detected, and where projectile and target are unpolarized can be written as [5]

$$
\begin{aligned}
\left(\frac{d \sigma}{d \Omega}\right)_{e l}=\sigma_{M} f_{r e c} & \left\{\frac{1}{1+\tau}\left(G_{C}^{N^{2}}\left(Q^{2}\right)+\tau G_{M}^{N^{2}}\left(Q^{2}\right)\right)\right. \\
& \left.+2 \tau \tan ^{2}\left(\frac{\Theta}{2}\right) G_{M}^{N}\left(Q^{2}\right)\right\} .
\end{aligned}
$$

This equation is known as Rosenbluth formula. Here, $G_{C}^{N}\left(Q^{2}\right)$ and $G_{M}^{N}\left(Q^{2}\right)$ are the charge monopole and magnetic dipole form factors of the nucleon, usually refered to as Sachs form factors [6]. The square of the four momentum transfer of the virtual photon [7] is denoted by $Q^{2}$ and $\tau=: Q^{2} /\left(4 M_{N}^{2}\right)$, where $M_{N}$ is the nucleon mass. Furthermore, $f_{\text {rec }}=\left(1+2\left(\epsilon_{i} / M_{N}\right) \sin ^{2}(\Theta / 2)\right)^{-1}$ is the nucleon recoil factor, where $\epsilon_{i}$ is the incident electron energy, and $\Theta$ is the electron scattering angle.

\footnotetext{
*published in: AIP Conf. Proc. 904 (2007) pg. 110.

${ }^{\dagger}$ Electronic address: alfons.buchmann@uni-tuebingen.de
}

The form factors in Eq.(1) can be determined from the measured cross section with the help of the Rosenbluth separation method [8]. Unlike the cross section, which contains coordinate frame dependent kinematical variables, the form factors depend only on the Lorentz invariant $Q^{2}$. They are therefore well suited for a comparison between experiment and theory. At $Q^{2}=0$ the Sachs form factors are normalized to the charge and magnetic dipole moment of the nucleon, i.e., $G_{C}^{p}(0)=1$ and $G_{M}^{p}(0)=\mu_{p}$ for the proton, and $G_{C}^{n}(0)=0$ and $G_{M}^{p}(0)=\mu_{n}$ for the neutron.

Until a few years ago, unpolarized scattering data analyzed with the Rosenbluth separation method provided ample evidence for a scaling law, i.e., for a wide range of momentum transfers both proton form factors, and the neutron magnetic form factor could be approximately described by a common dipole function $G_{D}\left(Q^{2}\right)$

$$
G_{C}^{p}\left(Q^{2}\right)=\frac{G_{M}^{p}}{\mu_{p}}\left(Q^{2}\right)=\frac{G_{M}^{n}}{\mu_{n}}\left(Q^{2}\right)=G_{D}\left(Q^{2}\right)
$$

where $G_{D}\left(Q^{2}\right)=\left(1+Q^{2} / \Lambda^{2}\right)^{-2}$, and $\Lambda^{2}$ is related to the spatial extension of the proton charge and magnetization densities. These equations suggest that at each point in space the local charge and magnetization densities in the proton are approximately equal, and that the magnetization density in the proton is equal to the magnetization density in the neutron. The neutron charge form factor has a different functional behavior, and will be discussed below. It has been shown that a scaling law as in Eq.(2) arises in constituent quark models if exchange currents between the quarks are neglected, and if the charge and magnetic form factors of constituent quarks are assumed to be equal [9].

More recent electron-proton experiments employing polarized electrons and measuring the polarization of the recoiling proton in coincidence with the scattered electron have led to very different results for the nucleon form factors [10-12]. Unlike the unpolarized elastic cross section of Eq.(1), which is proportional to an incoherent sum 

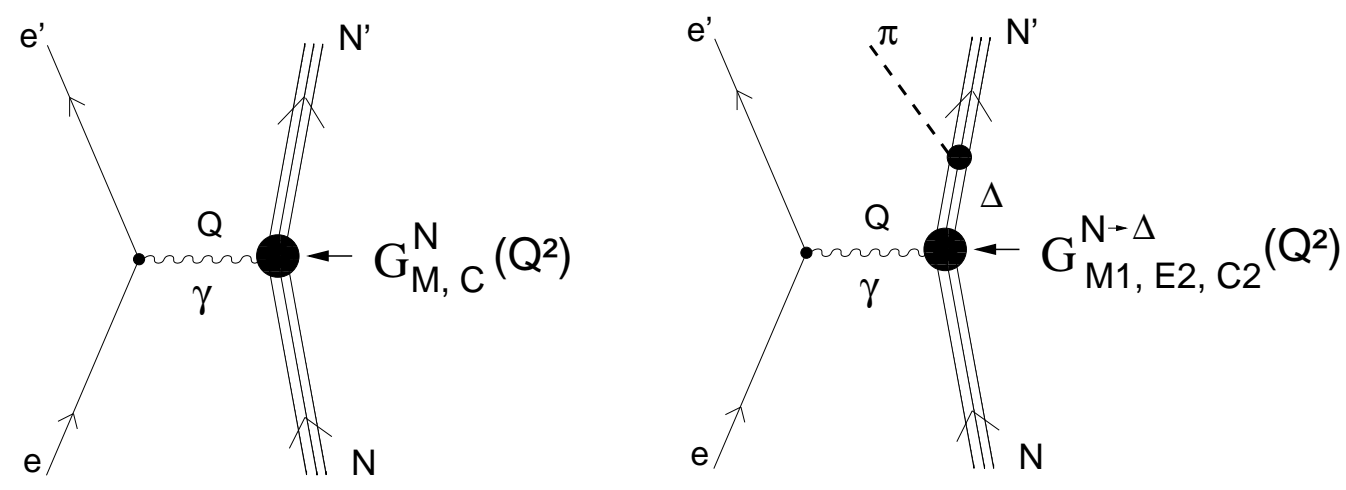

FIG. 1: Left: Probing of nucleon structure via elastic electron-nucleon scattering $e N \rightarrow e^{\prime} N^{\prime}$ involving the exchange of a single virtual photon $\gamma$ of momentum $Q$. The nucleon structure information is contained in the charge monopole form factor $G_{C}^{N}\left(Q^{2}\right)$ and magnetic dipole form factor $G_{M}^{N}\left(Q^{2}\right)$. Right: Inelastic electron-nucleon scattering $e N \rightarrow e^{\prime} \Delta$. The excitation of the $\Delta$ resonance is described by three electromagnetic transition form factors $G_{M 1}^{N \rightarrow \Delta}\left(Q^{2}\right), G_{E 2}^{N \rightarrow \Delta}\left(Q^{2}\right)$, and $G_{C 2}^{N \rightarrow \Delta}\left(Q^{2}\right)$. Relations between elastic and inelastic nucleon charge form factors provide information on the spatial shape of the nucleon.

of the squares of these form factors, the polarized cross section also contains interference terms of the two elastic form factors. The latter permit the extraction of more accurate results for the charge form factor compared to those obtained with Rosenbluth separation. For a review see Ref. [13]. At high momentum transfers the new experiments on the proton show large deviations from scaling between charge and magnetic form factors. This is indicative of substantial differences between the charge and magnetization distributions of the proton. Thus, the elastic form factors contain more information on nucleon structure than previously thought. In the following we suggest that the scaling law violation is a consequence of the proton's nonspherical charge distribution.

First evidence for a deviation of the nucleon's charge distribution from spherical symmetry came from studies of inelastic electron-nucleon scattering (Fig. 1) in which the $\Delta(1232)$ resonance with spin $3 / 2$ and isospin $3 / 2$ is excited. The $\Delta$ resonance is the lowest lying excited state of the nucleon $N(939)$ with the same quark content as the ground state. Parity invariance and angular momentum conservation restrict the electromagnetic $N \rightarrow \Delta$ excitation to magnetic dipole (M1), electric quadrupole (E2), and charge (or Coulomb) quadrupole (C2) transitions. In terms of transition multipole form factors, which are the inelastic counterparts of the elastic Sachs form factors in Eq.(1), the cross section for electroproduction of the $\Delta$ resonance is given by a generalized Rosenbluth formula

$$
\begin{gathered}
\left(\frac{d \sigma}{d \Omega}\right)_{\text {inel }}^{=\sigma_{M}} f_{\text {rec }} \frac{Q^{2}}{4 M_{N} M_{\Delta}} \frac{1}{1+\tilde{\tau}}\left\{\frac{Q^{2} M_{N}^{2}}{9}\left(G_{C 2}^{N \rightarrow \Delta}\right)^{2}\left(Q^{2}\right)\right. \\
\left.+\epsilon^{-1}\left(\left(G_{M 1}^{N \rightarrow \Delta}\right)^{2}\left(Q^{2}\right)+\frac{M_{N}^{2} \mathbf{q}^{2}}{18}\left(G_{E 2}^{N \rightarrow \Delta}\right)^{2}\left(Q^{2}\right)\right)\right\},(3)
\end{gathered}
$$

where $\epsilon=\left(1+2\left(M_{\Delta}^{2} / M_{N}^{2}\right)\left(|\mathbf{q}|^{2} / Q^{2}\right) \tan ^{2}(\Theta / 2)\right)^{-1}$ is the polarization of the transverse photons in the laboratory frame, and $\tilde{\tau}=: Q^{2} /\left(M_{N}+M_{\Delta}\right)^{2}$. The form factors are normalized to the transition magnetic moment $G_{M 1}^{N \rightarrow \Delta}(0)=\mu_{N \rightarrow \Delta}$ and quadrupole moment $G_{C 2}^{N \rightarrow \Delta}(0)=Q_{N \rightarrow \Delta}$. Cross section formulae analogous to Eq.(3) have been written by several authors [14, 15]. The generalized Sachs form factors in Eq.(3) are based on the same definition of the multipole operators [16] used for the elastic form factors in Eq.(1), and facilitate the comparison between elastic and inelastic nucleon form factors.

In the cross section of Eq.(3) the pions from the strong decay of the $\Delta$-resonance are not observed, only the scattered electrons are detected. As a result, the cross section formula involves only an incoherent sum of the squares of the multipole form factors, and the small quadrupole form factors cannot be reliably determined. On the other hand, if the nucleon or pion from the decay $\Delta \rightarrow N+\pi$ is detected in coincidence with the scattered electron, as depicted in Fig. 1, the corresponding cross section formula contains an interference term between the small charge quadrupole and the large magnetic dipole amplitudes. The contribution of the former to the cross section is thereby greatly enhanced, and can be more reliably determined. For a discussion of the coincidence cross section for pion electroproduction see Refs. [17].

At low momentum transfers, experiment shows that the $N \rightarrow \Delta$ excitation is predominantly an M1 transition, which can be interpreted as the spin and isospin flip of a single quark. The quadrupole amplitudes are only about $1 / 40$ of the dominant magnetic dipole amplitude. They can be interpreted as arising from a double spin flip involving two interacting quarks [18]. Despite their smallness, the $\mathrm{C} 2$ and E2 multipoles have been the focus of many recent experimental $[17,19-25]$ and theoretical works [26-32]. It becomes clear below that the transition quadrupole moments are nonzero only if the geometric shape of the nucleon deviates from spherical symmetry, and that from their sign and size information on the shape of the nucleon's charge distribution can be infered [33]. 
In this paper we show that further information on the geometrical shape of the nucleon can be obtained from the study of the relations between inelastic and elastic form factors. Generally, the geometric properties of the ground state of a physical system, in particular its size and shape, have a direct bearing on the eigenfrequencies and eigenmodes of its excitation spectrum. Conversely, knowledge of the eigenfrequencies and excitation modes of a system enables us to draw certain conclusions concerning its size and shape. In the case of the nucleon, we have suggested that the existence of a quadrupole excitation mode of the $\Delta$-resonance is closely related to a quadrupole deformation of the nucleon's charge distribution as reflected by a positive intrinsic quadrupole moment [33] and an intrinsic charge quadrupole form factor [34]. The latter has observable consequences for the elastic nucleon form factors. To show this, we decompose in chapter IV the proton charge form factor into a spherically symmetric term and a nonspherical intrinsic quadrupole term, where the latter is given by the $N \rightarrow \Delta$ charge quadrupole transition form factor. Before doing this, we review in chapter II the empirical information on the relations between the elastic nucleon and inelastic $N \rightarrow \Delta$ electromagnetic form factors, and how these can be understood in terms of strong interaction symmetries, in particular spin-flavor symmetry.

\section{ELECTROMAGNETIC $N \rightarrow \Delta$ TRANSITION AND SPIN-FLAVOR SYMMETRY}

\section{A. Strong interaction symmetries}

It is well known that invariance of the strong interaction under $\mathrm{SU}(2)$ isospin transformations leads to isospin conservation and the appearance of degenerate hadron multiplets with fixed isospin, in which individual hadrons have different charges but nearly the same mass. Moreover, also other properties of the particles within an isospin multiplet are closely related by the underlying symmetry group. This remains true even if the symmetry is broken. As discussed below, the group algebra guarantees that symmetry breaking occurs according to a well defined scheme, with the result that its consequences for different members of an isospin multiplet are related by the Wigner-Eckart theorem, i.e., the matrix elements of a given isospin breaking operator evaluated for different members of an isospin multiplet differ only by a Clebsch-Gordan coefficient.

But strong interactions are invariant under a higher symmetry than isospin. Flavor $\mathrm{SU}(3)$ symmetry ties together isospin multiplets with different isospin and different strangeness to larger multiplets with the same spin and parity, e.g., octets and decuplets [35]. Even though mass differences between different isospin multiplets within the baryon flavor octet and decuplet are of the order of $100 \mathrm{MeV}$, the inclusion of the relevant symmetry breaking operators leads to a number of re- markable predictions, such as the Gell-Mann-Okubo relation between octet baryon masses, or the equal spacing rule between the masses of the isospin multiplets in the baryon decuplet, which are in good agreement with experiment.

An even higher strong interaction symmetry than $\mathrm{SU}(3)$ flavor is $\mathrm{SU}(6)$ spin-flavor, which unites the spin $1 / 2$, flavor octet baryons $(2 \times 8$ states $)$, among them the familiar proton and neutron, and the spin $3 / 2$, flavor decuplet baryons $(4 \times 10$ states $)$, among them the four $\Delta$ states into a common 56-dimensional supermultiplet [3638]. More formally, the SU(6) ground state supermultiplet can be decomposed into irreducible representations of the flavor and spin subgroups as $\mathbf{5 6}=(\mathbf{8}, \mathbf{2})+(\mathbf{1 0}, \mathbf{4})$, where the first and second label refer to the $\mathrm{SU}(3)$ and $\mathrm{SU}(2)$ dimensions respectively. There are numerous successful predictions based on $\mathrm{SU}(6)$ spin-flavor symmetry. For example, SU(3) flavor symmetry alone does not suffice to uniquely determine the ratio of proton and neutron magnetic moments, whereas $\mathrm{SU}(6)$ spin-flavor symmetry leads to the prediction [38] $\mu_{p} / \mu_{n}=-3 / 2$, in excellent agreement with the experimental result 1.46. Another example for the predictive power of $\mathrm{SU}(6)$ spin-flavor symmetry is the Gürsey-Radicati mass formula [36], which explains why the Gell-Mann Okubo mass formula works so well for both octet and decuplet baryons with the same numerical coefficients. Without SU(6) it would remain a mystery why the violation of SU(3) symmetry in the baryon octet is the same as in the baryon decuplet. This fact is not explained in $\mathrm{SU}(3)$.

Thus, $\mathrm{SU}(6)$ is an excellent symmetry in baryon physics, and the question arises whether it is a symmetry of quantum chromodynamics. This is indeed the case. In an $1 / N_{c}$ expansion of QCD, where $N_{c}$ denotes the number of colors, it has been shown that QCD possesses a spin-flavor symmetry, which is exact in the large $N_{c}$ limit $[39,40]$, and that for finite $N_{c}$ spin-flavor symmetry breaking operators can be classified according to the powers of $1 / N_{c}$ associated with them. It turns out that higher orders of spin-flavor symmetry breaking are suppressed by correspondingly higher powers of $1 / N_{c}$. For example, second and third order $\mathrm{SU}(6)$ symmetry breaking described by two- and three-quark operators are suppressed by $1 / N_{c}$ and $1 / N_{c}^{2}$ respectively, compared to the first order symmetry breaking due to one-quark operators. As a result, one obtains a rigorous perturbative expansion scheme for QCD processes that works at all energy scales, and which furthermore provides a connection between broken $\mathrm{SU}(6)$ spin-flavor symmetry and the underlying quark-gluon dynamics [41]. This allows us to put certain model results on a solid theoretical foundation. In the next section, we employ SU(6) symmetry and its breaking to study the relations between inelastic $N \rightarrow \Delta$ transition form factors and elastic nucleon form factors. 


\section{B. Electromagnetic $N \rightarrow \Delta$ transition and nucleon form factors}

Because the $N$ and $\Delta$ belong to the same 56dimensional ground state multiplet of the SU(6) spinflavor group their properties are related. In particular, the electromagnetic $N \rightarrow \Delta$ transition form factors are related to the electromagnetic elastic form factors of the nucleon. In fact, the $\mathrm{SU}(6)$ relation between the magnetic dipole transition form factor $G_{M 1}^{N \rightarrow \Delta}\left(Q^{2}\right)$ and the elastic neutron magnetic form factor $G_{M}^{n}\left(Q^{2}\right)$ has been known for some time [38]

$$
G_{M 1}^{N \rightarrow \Delta}\left(Q^{2}\right)=-\sqrt{2} G_{M}^{n}\left(Q^{2}\right)
$$

At $Q^{2}=0$, both form factors are normalized to their magnetic dipole moments $\mu_{N \rightarrow \Delta}$ and $\mu_{n}$

$$
\mu_{N \rightarrow \Delta}=-\sqrt{2} \mu_{n}
$$

These relations also hold when second order SU(6) symmetry breaking operators are included [42]. They have also been derived in the quark model with two-quark currents [43], and are violated only by three-quark currents [44] or third order $\mathrm{SU}(6)$ symmetry breaking operators [45]. The latter are suppressed by a factor $1 / N_{c}^{2}$ with respect to the leading term so that these relations are valid in good approximation.

The other relation between the charge quadrupole transition form factor $G_{C 2}^{N \rightarrow \Delta}\left(Q^{2}\right)$ and the elastic neutron charge form factor $G_{C}^{n}\left(Q^{2}\right)$

$$
G_{C 2}^{N \rightarrow \Delta}\left(Q^{2}\right)=-\frac{3 \sqrt{2}}{Q^{2}} G_{C}^{n}\left(Q^{2}\right)
$$

has been found only quite recently within a quark model that includes in addition to the usual single quark electromagnetic current also two-body exchange currents associated with the quark-quark interaction [43]. In the $Q \rightarrow 0$ limit, Eq.(6) reduces to a relation [18] between the transition quadrupole moment $Q_{N \rightarrow \Delta}$ and the neutron charge radius $r_{n}^{2}$

$$
Q_{N \rightarrow \Delta}=\frac{1}{\sqrt{2}} r_{n}^{2}
$$

which is in good agreement with recent extractions of $Q_{N \rightarrow \Delta}$ from the data $[27,46]$. Also at low momentum transfers, Eq.(6) is satisfied by the data [47]. More recently, it has been shown that the validity of Eq.(6) is not confined to low momentum transfers but extends into the $\mathrm{GeV}$ region [48].

Experimental results are often given for the $C 2 / M 1$ ratio, which is defined in terms of the $N \rightarrow \Delta$ transition form factors times a kinematical factor [14, 49]

$$
\frac{C 2}{M 1}\left(Q^{2}\right)=: \frac{|\mathbf{q}| M_{N}}{6} \frac{G_{C 2}^{N \rightarrow \Delta}\left(Q^{2}\right)}{G_{M 1}^{N \rightarrow \Delta}\left(Q^{2}\right)},
$$

where $M_{N}$ is the nucleon mass and $|\mathbf{q}|$ is the threemomentum transfer of the virtual photon in the $\gamma N$ center of mass frame [50]. Inserting the above form factor relations of Eq.(4) and Eq.(6), the $C 2 / M 1$ ratio can be expressed as the product of $G_{C}^{n} / G_{M}^{n}$ and a factor

$$
\frac{C 2}{M 1}\left(Q^{2}\right)=\frac{|\mathbf{q}|}{Q} \frac{M_{N}}{2 Q} \frac{G_{C}^{n}\left(Q^{2}\right)}{G_{M}^{n}\left(Q^{2}\right)}=: \mathcal{R}_{n}\left(Q^{2}\right) .
$$

We abbreviate this product as $\mathcal{R}_{n}\left(Q^{2}\right)$. Thus, the inelastic $N \rightarrow \Delta$ and the elastic neutron form factor ratios are related. The theoretical uncertainty of this relation is mainly due to third order $\mathrm{SU}(6)$ symmetry breaking terms (three-quark currents) omitted in Eq.(4). We estimate it to be of order $1 / N_{c}^{2}$ or $10 \%$ (slightly increasing the predicted $C 2 / M 1$ ratio).

For convenience, we calculate the ratio $\mathcal{R}_{n}$ of Eq.(9) using for the numerator a two-parameter fit [51] of the $G_{C}^{n}$ data and for the denominator the dipole fit $G_{D}$ for $G_{M}^{n}$, i.e.,

$$
\begin{aligned}
G_{C}^{n}\left(Q^{2}\right) & =-\mu_{n} \frac{a \tau}{1+d \tau} G_{D}\left(Q^{2}\right), \\
G_{M}^{n}\left(Q^{2}\right) & =\mu_{n} G_{D}\left(Q^{2}\right),
\end{aligned}
$$

where $G_{D}$ is the dipole form factor of Eq.(2) with $\Lambda^{2}=$ $0.71 \mathrm{GeV}^{2}$. The $C 2 / M 1$ ratio is then given in terms of the parameters $a$ and $d$, which have been determined from the lowest moments $\left(r_{n}^{2}\right.$ and $\left.r_{n}^{4}\right)$ of the experimental neutron charge form factor [47]. In Fig. 2 we plot $\mathcal{R}_{n}$ calculated from $G_{C}^{n} / G_{M}^{n}$ data using Eq.(10) and compare with $C 2 / M 1$ data from various pion electroproduction experiments [20, 25, 53-56]. The solid, dashed-dotted, and double dashed-dotted lines correspond to decreasing values of the Galster parameter $d$. The chosen parameterization is most likely too restrictive at high $Q^{2}$, where it would be preferable to compare directly with measured neutron form factor data.

Next, we evaluate Eq.(9) for very low and very high $Q^{2}$. In the real photon limit $Q \rightarrow 0$ we obtain [50]

$$
\frac{C 2}{M 1}(0)=-\frac{M_{\Delta}^{2}-M_{N}^{2}}{2 M_{\Delta}} \frac{M_{N}}{12} \frac{r_{n}^{2}}{\mu_{n}}=-0.031
$$

in good agreement with the experimental $E 2 / M 1$ ratio obtained from pion photoproduction by different groups $[46,52,58,59]$. This result explains the experimental value for the $C 2 / M 1$ ratio in terms of the charge radius and the magnetic moment of the neutron. We understand therefore why $C 2 / M 1(0)=-0.03$. For $Q^{2} \rightarrow \infty$ we obtain using Eq.(10)

$$
\mathcal{R}_{n}\left(Q^{2} \rightarrow \infty\right)=\frac{1}{4} \frac{M_{N}}{M_{\Delta}}\left(-\frac{a}{d}\right),
$$

which ranges between -0.06 and -0.21 depending on the parameter $d$. Thus, we see that the $C 2 / M 1$ ratio asymptotically approaches a small negative constant determined by the neutron structure parameters $a$ and $d$. 


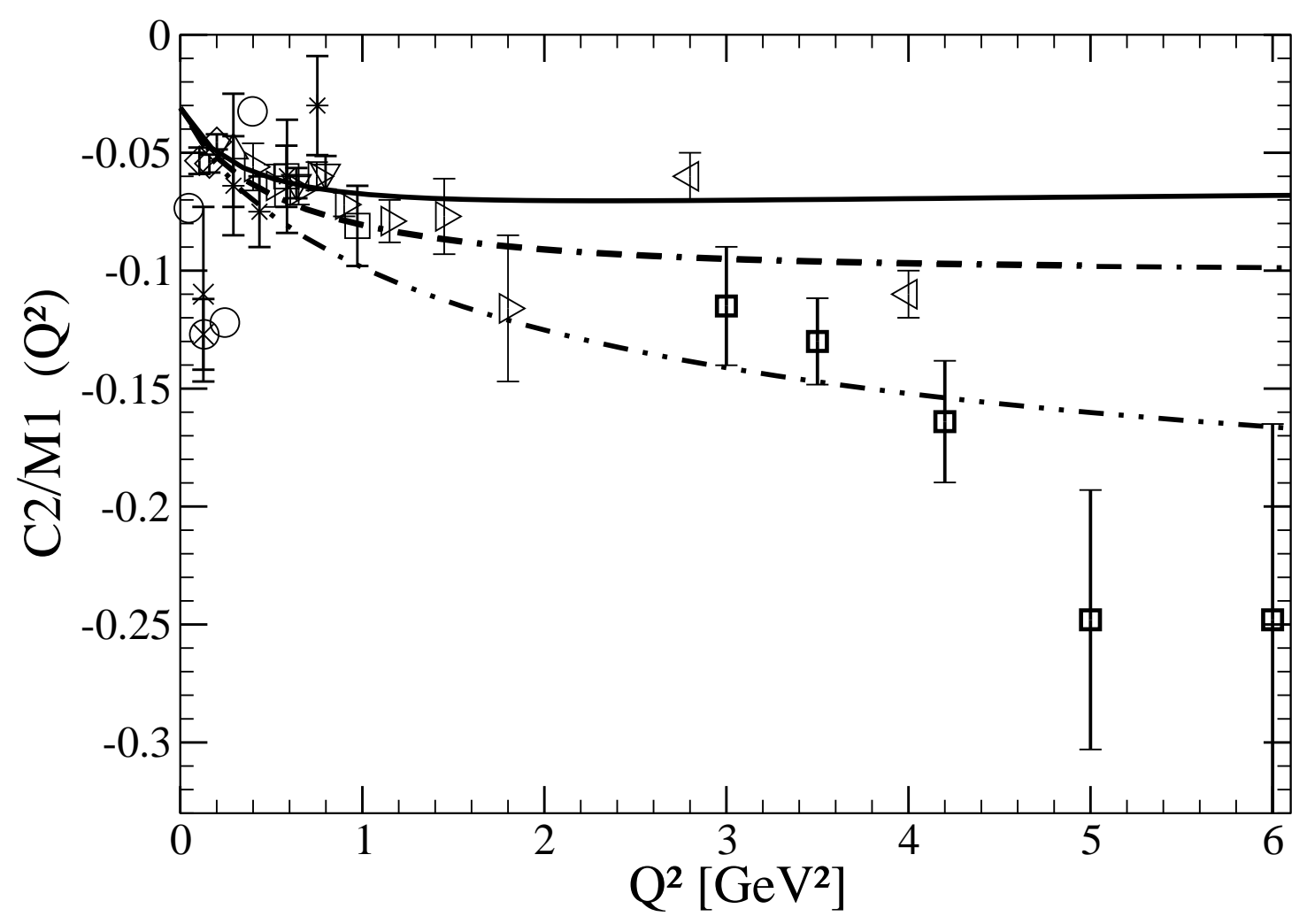

FIG. 2: The ratio $\mathcal{R}_{n}$ of Eq.(9) calculated from a two-parameter fit of elastic neutron form factor data according to Eq.(10). Solid curve for $a=0.9$ and $d=2.8$, dashed-dotted curve for $a=0.9$ and $d=1.75$ [47], and double-dotted curve for $a=0.9$ and $d=0.8$. This is compared with experimental results for the $C 2 / M 1$ ratio extracted from pion electroproduction cross sections $[20,25,53-57]$.

This is in qualitative agreement with expectations from PQCD [26] modulo logarithmic corrections.

We conclude that the two data sets, which were thought to be quite independent of each other, satisfy the proposed relation Eq.(9) within experimental uncertainties for momentum transfers between 0 and $3 \mathrm{GeV}^{2}$, which in turn suggests that Eq.(6) is well satisfied in nature. As to the physical interpretation of this relation, we learn from Eq.(7) that the small deviation of $r_{n}^{2}$ from zero and the deviation of the nucleon's geometric shape from spherical symmetry as manifested in a nonzero $Q_{N \rightarrow \Delta}$ are closely related aspects of nucleon structure. Both phenomena have their origin in a nonspherical cloud of quark-antiquark pairs in the nucleon [33], which are effectively described as exchange currents between quarks [60]. This interpretation continues to hold for finite momentum transfers as suggested by the agreement of Eq.(9) with the data. We elaborate on this in chapter IV. On a more abstract level, one can understand this relation in terms of the under- lying spin-flavor symmetry of QCD and its breaking by spin-dependent exchange currents.

\section{SU(6) spin-flavor symmetry analysis}

We start from the observation that the $N(939)$ and $\Delta(1232)$ are members of the same $\mathbf{5 6}$ dimensional ground state multiplet of $\mathrm{SU}(6)$ spin-flavor symmetry. If this symmetry were exact, $N$ and $\Delta$ baryons would have the same mass, and the form factors $G_{C}^{n}\left(Q^{2}\right)$ and $G_{C 2}^{N \rightarrow \Delta}\left(Q^{2}\right)$ would be exactly zero. Spin-dependent two-body potentials in the Hamiltonian break $\mathrm{SU}(6)$ symmetry and lift the degeneracy between $N$ and $\Delta$ masses [61]. Similarly, spin-dependent two-body terms in the charge operator break $\mathrm{SU}(6)$ symmetry and lead to nonzero neutron and $N \rightarrow \Delta$ charge form factors, which are related as in Eq.(6) because the group algebra connects the breaking of the symmetry in $G_{C}^{n}$ to the symmetry breaking in $G_{C 2}^{N \rightarrow \Delta}$. 
This can be most easily seen from a multipole expansion of the relevant two-quark charge density $\rho_{[2]}$ in spinflavor space

$\rho_{[2]}=-B \sum_{i \neq j}^{3} e_{i}\left[2 \boldsymbol{\sigma}_{i} \cdot \boldsymbol{\sigma}_{j}-\left(3 \sigma_{i z} \sigma_{j z}-\boldsymbol{\sigma}_{i} \cdot \boldsymbol{\sigma}_{j}\right)\right]=2 \mathcal{S}-\mathcal{T}$,

where $B$ represents the color and orbital degrees of freedom, and $e_{i}=\left(1+3 \tau_{3 i}\right) / 6$ is the quark charge. Here, $\boldsymbol{\sigma}_{i}$ and $\boldsymbol{\tau}_{i}$ are the spin and isospin Pauli matrices of the i-th quark. The subscript [2] indicates that this operator acts on two qarks at a time. As will become clearer below, to first order flavor (isospin) breaking, Eq.(13) represents the most general two-body charge operator in spin-flavor (isospin) space. The factors multiplying the spin scalar $\mathcal{S}=-B \sum_{i \neq j} e_{i} \boldsymbol{\sigma}_{i} \cdot \boldsymbol{\sigma}_{j}$ and spin tensor $\mathcal{T}=-B \sum_{i \neq j} e_{i}\left(3 \sigma_{i z} \sigma_{j z}-\boldsymbol{\sigma}_{i} \cdot \boldsymbol{\sigma}_{j}\right)$ have a fixed ratio. In the quark model this arises because both operator structures originate from the same diagram, e.g., the one gluon-exchange current [60]. But the same relation Eq.(6) is obtained if we use one-pion exchange currents or a combination of one-gluon and one-pion exchange currents. An evaluation of Eq.(13) between $N$ and $\Delta$ spin-flavor wave functions leads straightforwardly to the following relations [18]

$$
Q_{N \rightarrow \Delta}=\frac{1}{\sqrt{2}} r_{n}^{2}, \quad Q_{\Delta}=e_{\Delta} r_{n}^{2},
$$

where $e_{\Delta}$ denotes the charge of the $\Delta$ state.

More generally, one can understand the relation between the neutron charge radius and the $N \rightarrow \Delta$ quadrupole moment in terms of a group-theoretical analysis without reference to specific dynamical assumptions, such as one-gluon exchange. A basic assumption in a group-theoretical analysis is that operators and states have definite transformation properties, i.e., they transform according to certain irreducible representations of the underlying symmetry group. A general matrix element $\mathcal{M}$ of an operator $\Omega_{R}$ evaluated between states belonging to the $\mathbf{5 6}$ dimensional representation of $\mathrm{SU}(6)$ then reads

$$
\mathcal{M}=\left\langle 56\left|\Omega_{R}\right| 56\right\rangle,
$$

where $R$ is the dimension of the irreducible representation according to which the considered operator transforms. In the following, we denote the irreducible representations of the states and operators by their dimension. An allowed symmetry breaking operator $\Omega_{R}$ acting on the ground state multiplet must then transform according to one of the irreducible representations $R$ found in the product [37]

$$
56 \times 56=1+35+405+2695 .
$$

Operators transforming according to other SU(6) representations not contained in this product will lead to vanishing matrix elements when evaluated between states belonging to the 56. On the right-hand side, the $\mathbf{1}$ dimensional representation is associated with a zero-body operator (constant), and the 35, 405, and 2695, are respectively connected with one-, two-, and three-body operators. It is important to note that one-body operators transforming according to a $\mathbf{3 5}$ dimensional representation of $\mathrm{SU}(6)$ do not lift the degeneracy between $N$ and $\Delta$ masses, and do not generate nonvanishing baryon quadrupole moments and neutral baryon charge radii. For these observables, first order SU(6) symmetry breaking operators do not suffice to obtain results in agreement with experiment.

Higher order symmetry breaking operators can be constructed from direct products of one-body operators. Consequently, a general two-body spin-flavor operator transforms according to the irreducible representations obtained from the direct product $\mathbf{3 5} \times \mathbf{3 5}$ as follows [37]

$$
35 \times 35=1+35+35+189+280+2 \overline{8} 0+405 .
$$

Two-body operators transforming according to the $\mathbf{1}$ or 35 dimensional SU(6) representation can be reduced to constants and one-body operators, so that only the four higher dimensional representations on the right hand side of Eq.(17) remain. However, according to Eq.(16) only the $\mathbf{4 0 5}$ appears in the direct product $\overline{\mathbf{5 6}} \times \mathbf{5 6}$. Therefore, within the $\mathbf{5 6}$ an allowed two-body operator must necessarily transform according to the $\mathbf{4 0 5}$ dimensional representation of $\mathrm{SU}(6)$.

Next, we show that the two-body operators appearing in Eq.(13) are components of this general SU(6) tensor. This can be seen from a decomposition of the tensor operator $\Omega_{405}$ into tensors with definite transformation properties with respect to the flavor and spin subgroups of $\mathrm{SU}(6)$

$$
\begin{aligned}
\mathbf{4 0 5} & =(\mathbf{1}, \mathbf{1})+(\mathbf{8}, \mathbf{1})+(\mathbf{2 7}, \mathbf{1}) \\
& +2(\mathbf{8}, \mathbf{3})+(\mathbf{1 0}, \mathbf{3})+(\mathbf{1 0}, 3)(\mathbf{2 7}, \mathbf{3}) \\
& +(\mathbf{1}, \mathbf{5})+(\mathbf{8}, \mathbf{5})+(\mathbf{2 7}, \mathbf{5}),
\end{aligned}
$$

where the first and second entry in the parentheses refers to the dimensions of the $\mathrm{SU}(3)$ and $\mathrm{SU}(2)$ representations respectively [62]. Because we deal with a charge operator, and the quark charge $e_{i}$ transforms as a flavor $\mathbf{8}$, we confine ourselves to operators transforming as octets, i.e., to first order symmetry breaking in flavor space. Furthermore, for Coulomb multipoles, allowed operators are restricted to $\mathrm{SU}(2)$ tensors of even rank. Thus, the spin scalar operator $\mathcal{S}$ transforms as the $(\mathbf{8}, \mathbf{1})$ and the spin tensor operator $\mathcal{T}$ as $(\mathbf{8}, \mathbf{5})$ and both are united in a common $\mathrm{SU}(6)$ representation with dimension $\mathbf{4 0 5}$. In other words, the two-body operators $\mathcal{S}$ and $\mathcal{T}$ in Eq.(13) are the only allowed structures on the right hand side of Eq.(18), and are recognized here as different components of a common 405 dimensional tensor operator $\Omega_{\mathbf{4 0 5}}$.

The matrix elements of this operator evaluated between the $\mathbf{5 6}$ multiplet can be factorized according to a generalized Wigner-Eckart theorem into a common reduced matrix element which is the same for the entire 
multiplet and various SU(6) Clebsch-Gordan coefficients. The latter provide relations between the matrix elements of different components of the $\Omega_{\mathbf{4 0 5}}$ and the 56. These group-theoretical arguments explain why the two operators $\mathcal{S}$ and $\mathcal{T}$ have a fixed ratio and why their matrix elements within the $\mathbf{5 6}$ are related. A derivation of Eq.(6) requires explicit $\mathrm{SU}(6)$ tensor representations of the operators and states.

In order to further explore the range of validity of this relation without having to use an explicit tensor notation for the $\mathbf{4 0 5}$ and $\mathbf{5 6}$, the neutron charge radius and $N \rightarrow$ $\Delta$ transition quadrupole moment have been evaluated in an $1 / N_{c}$ expansion [30] including third order $\mathrm{SU}(6)$ symmetry breaking due to three-body operators $\rho_{[3]}$. The following expression has been found:

$$
Q_{N \rightarrow \Delta}=\frac{1}{\sqrt{2}} r_{n}^{2}\left(\frac{N_{c}}{N_{c}+3} \sqrt{\frac{N_{c}+5}{N_{c}-1}}\right) .
$$

It is interesting that this more general relation is equivalent to Eq.(7) for the physical $N_{c}=3$ case and for $N_{c} \rightarrow \infty$.We conclude that Eq.(7) and its generalization to finite momentum transfers in Eq.(6) are of more general validity than Eq.(4) because they also hold in a theory [30], which includes spin-dependent three-quark terms in the charge density, and for an arbitrary odd number of colors $N_{c}>1$. In summary, the above arguments suggest that the relation between the neutron charge and $N \rightarrow \Delta$ transition form factors should be well satisfied in nature.

\section{GEOMETRIC SHAPE OF THE NUCLEON}

We have already mentioned that the relation between the elastic and inelastic $N \rightarrow \Delta$ charge form factors has implications for the shape of the nucleon. The information on the shape is contained in higher multipole moments of the nucleon charge distribution in particular its quadrupole moment. In this context it is important to pay attention to the coordinate frame dependence of these higher multipole moments. From classical electrodynamics we know that higher multipoles depend on the choice of origin and the orientation of the coordinate axes.

\section{A. Spectroscopic and intrinsic quadrupole moments}

In ref. [33] we have drawn attention to the fact that in order to make statements concerning the shape of the nucleon, one has to determine its intrinsic quadrupole moment. The intrinsic quadrupole moment of a nucleus

$$
Q_{0}=\int d^{3} r \rho(\mathbf{r})\left(3 z^{2}-r^{2}\right)
$$

is defined with respect to the body-fixed frame. If the charge density is concentrated along the $z$-direction (symmetry axis of the particle), the term proportional to $3 z^{2}$ dominates, $Q_{0}$ is positive, and the particle is prolate (cigar-shaped). If the charge density is concentrated in the equatorial plane perpendicular to $z$, the term proportional to $r^{2}$ prevails, $Q_{0}$ is negative, and the particle is oblate (pancake-shaped).

The intrinsic quadrupole moment $Q_{0}$ must be distinguished from the spectroscopic quadrupole moment $Q$ measured in the laboratory frame. A simple example will illustrate this point. Suppose one has determined the quadrupole moment $Q_{0}$ of a classical charge distribution $\rho(\mathbf{r})$ with symmetry axis $z$ and angular momentum $J$ in the body-fixed frame according to Eq.(20). Then, the quadrupole moment of the same charge distribution with respect to the laboratory frame is given by

$$
\begin{aligned}
Q=P_{2}(\cos \theta) Q_{0} & =\frac{1}{2}\left(3 \cos ^{2}(\theta)-1\right) Q_{0} \\
& =\left(\frac{3 J_{z^{\prime}}{ }^{2}-J(J+1)}{2 J(J+1)}\right) Q_{0}
\end{aligned}
$$

where $\theta$ is the angle between the body-fixed $z$ and the laboratory frame $z^{\prime}$ axes, and $P_{2}$ is the second Legendre polynomial. The latter arises when tranforming the spherical harmonic of rank 2 in $Q_{0}$ from body-fixed to laboratory coordinates. The third equality in Eq.(21) is obtained when $\cos (\theta)$ is expressed in terms of the spin projection $J_{z^{\prime}}$ on the laboratory frame $z^{\prime}$-axis and the total spin $J$ of the system as $\cos (\theta)=J_{z^{\prime}} / \sqrt{J^{2}}$ (see Fig. 3).

Thus, in the laboratory one does not measure the intrinsic quadrupole moment directly but only its projection onto the $z^{\prime}$ axis. In the quantum mechanical analogue of Eq.(21) the denominator of the projection factor is changed into $(2 J+3)(J+1)[63]$. The projection factor shows that $J=0$ and $J=1 / 2$ systems have vanishing spectroscopic quadrupole moments even though they may be deformed and their intrinsic quadrupole moments are nonzero. For a $J=0$ nucleus this is intuitively clear because it does not have a preferred direction in space. All directions are equally probable so that in the laboratory frame a spherically symmetric charge distribution is observed. A similar argument holds for the $J=1 / 2$ proton, which does not have a spectroscopic quadrupole moment and which will always appear spherically symmetric in the laboratory frame. However, the nucleon can have and does have an intrinsic quadrupole moment $Q_{0}$. It is the latter which is related to the geometric shape of the nucleon.

\section{B. Intrinsic quadrupole moment of the nucleon}

To obtain information on the geometric shape of the nucleon we have used different models to estimate its intrinsic quadrupole moment [33]. For example, in the quark model we found

$$
Q_{0}^{p}=-Q_{0}^{\Delta^{+}}=-r_{n}^{2}
$$




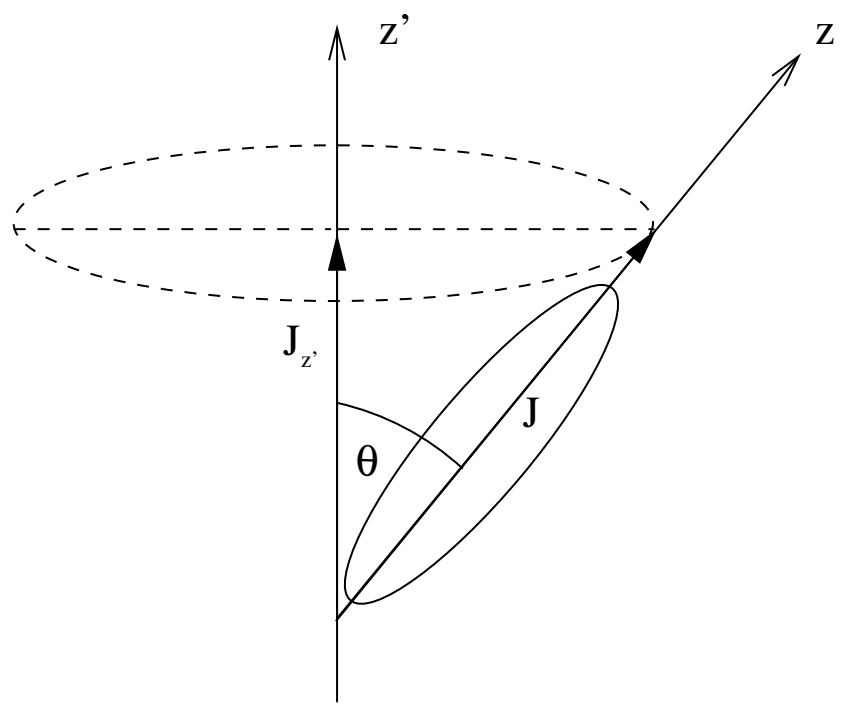

FIG. 3: Precession of a deformed charge distribution with intrinsic symmetry axis $z$ around the laboratory frame $z^{\prime}$ axis. The transformation from the body-fixed to the laboratory frame gives rise to a projection factor $P_{2}(\cos (\theta))=$ $1 / 2\left(3 \cos ^{2}(\theta)-1\right)$ relating spectroscopic quadrupole moment $Q$ and intrinsic quadrupole moment $Q_{0}$. Only the latter pertains to the shape of the system.

i.e., the intrinsic quadrupole moment of the proton is equal to the negative of the neutron charge radius and therefore positive, whereas the $\Delta^{+}$has a negative intrinsic quadrupole moment that is equal to its spectroscopic quadrupole moment. This corresponds to a prolate proton and an oblate $\Delta^{+}$deformation. A comparison of Eq.(22) with Eq.(7) shows that the intrinsic quadrupole moments of the $N$ and $\Delta$ are only then nonzero if the transition quadrupole moment $Q_{N \rightarrow \Delta}$ is nonzero.

In the quark model, the positive intrinsic quadrupole moment of the proton is obtained by evaluating the twoquark charge quadrupole operator

$$
Q_{[2]}=-B \sum_{i \neq j}^{3} e_{i}\left(3 \sigma_{i z} \sigma_{j z}-\boldsymbol{\sigma}_{i} \cdot \boldsymbol{\sigma}_{j}\right)
$$

between quark model wave functions in which the Clebsch-Gordan coefficients that express the coupling of the spin 1 diquark to the third quark have been renormalized to 1 . The reason for doing this is the following. Although the two spin 1 diquarks in the proton ( $u u$ and $u d$ ) have nonvanishing quadrupole moments, the coupling of the diquark spin to the spin $1 / 2$ of the third quark prevents these quadrupole moments from being observed. After renormalization of the spin coupling coefficients, the contributions of the spin 1 diquarks in the $M=0$ and $M=1$ substates no longer cancel each other but the $M=0$ component prevails. This results in an overall positive quadrupole moment, which we identify with the intrinsic quadrupole moment of the proton $Q_{0}^{p}$. A similar calculation for the neutron shows that its intrinsic quadrupole moment is also positive.
A simple way to picture these results is the following is to recall that the two-quark spin-spin operators are repulsive between quark pairs with spin 1 . In the proton they push the up quarks further apart than an up-down quark pair. This results in an elongated (prolate) charge distribution with the down quark in the middle. Likewise, in the neutron we have an elongated (prolate) charge distribution with the up quark in the middle and the negative down quarks at the periphery leading to a negative neutron charge radius. In the $\Delta^{+}$with only spin 1 quark pairs there is no asymmetry between up-down and up-up pairs. This corresponds to an equilateral triangle (oblate) configuration of its constituent quarks. However, this picture is an effective valence quark description of nucleon dynamics. In reality, the valence quarks are nearly spherically distributed, and the deformation of the nucleon's charge density resides in the quark-antiquark pairs, which are the physical origin of the two-quark operators in Eq.(23). In summary, the quark model with two-body exchange currents shows that the negative spectroscopic quadrupole moments of the $\Delta$ and the $N \rightarrow \Delta$ transition, and the negative neutron charge radius are different manifestations of a prolate intrinsic charge distribution of the nucleon.

A quite similar result suggesting a connection between the neutron charge radius $r_{n}^{2}$ and the intrinsic quadrupole moment of the proton $Q_{0}^{p}$ is also obtained in the pion cloud model (see Fig. 4). In this model, the nucleon consists of a spherically symmetric bare nucleon (quark core) surrounded by a pion with orbital angular momentum $l=1$.

For example, the physical proton with spin up, denoted by $|p \uparrow\rangle$, is a coherent superposition of three different terms: (i) a spherical quark core contribution with spin $1 / 2$, called a bare proton $p^{\prime}$; (ii) a bare $p^{\prime}$ surrounded by a neutral pion cloud, and (iii) a bare neutron $n^{\prime}$ surrounded by a positively charged pion cloud [64]. In the last two terms the spin(isospin) of the bare proton and of the pion cloud are coupled to total spin and isospin of the physical proton. Similarly, the physical $\Delta^{+}$is described as a superposition of a spherical quark core term with spin $3 / 2$, called a bare $\Delta^{+^{\prime}}$, a bare $p^{\prime}$ surrounded by a $\pi^{0}$ cloud, and a bare $n^{\prime}$ surrounded by a $\pi^{+}$cloud. In each term, the spin/isospin of the quark core and pion cloud are coupled to the total spin and isospin of the physical $\Delta^{+}$. We then write:

$$
\begin{aligned}
|p \uparrow\rangle & =\alpha\left|p^{\prime} \uparrow\right\rangle+\beta \frac{1}{3}\left(\left|p^{\prime} \uparrow \pi^{0} Y_{0}^{1}\right\rangle-\sqrt{2}\left|p^{\prime} \downarrow \pi^{0} Y_{1}^{1}\right\rangle\right. \\
& \left.-\sqrt{2}\left|n^{\prime} \uparrow \pi^{+} Y_{0}^{1}\right\rangle+2\left|n^{\prime} \downarrow \pi^{+} Y_{1}^{1}\right\rangle\right), \\
\left|\Delta^{+} \uparrow\right\rangle & =\alpha^{\prime}\left|\Delta^{+^{\prime}} \uparrow\right\rangle+\beta^{\prime} \frac{1}{3}\left(2\left|p^{\prime} \uparrow \pi^{0} Y_{0}^{1}\right\rangle+\sqrt{2}\left|p^{\prime} \downarrow \pi^{0} Y_{1}^{1}\right\rangle\right. \\
& \left.+\sqrt{2}\left|n^{\prime} \uparrow \pi^{+} Y_{0}^{1}\right\rangle+\left|n^{\prime} \downarrow \pi^{+} Y_{1}^{1}\right\rangle\right),
\end{aligned}
$$

where $\beta$ and $\beta^{\prime}$ describe the amount of pion admixture in the $N$ and $\Delta$ wave functions. These amplitudes satisfy the normalization conditions $\alpha^{2}+\beta^{2}=\alpha^{\prime 2}+\beta^{\prime 2}=1$, 
so that we have only two unknows $\beta$ and $\beta^{\prime}$. The corresponding wave functions for the neutron and $\Delta^{0}$ are obtained by isospin rotation [64]. Here, $Y_{0}^{1}$ and $Y_{1}^{1}$ are spherical harmonics of rank 1 describing the orbital angular momentum wave functions of the pion. Because the pion moves predominantly in a $p$-wave, the charge distributions of the nucleon and $\Delta$ deviate from spherical symmetry, even if the bare nucleon and bare $\Delta$ wave functions are spherical.

The quadrupole operator to be used in connection with these states is

$$
\hat{Q}=\hat{Q}_{\pi}=e_{\pi} \sqrt{\frac{16 \pi}{5}} r_{\pi}^{2} Y_{0}^{2}\left(\hat{\mathbf{r}}_{\pi}\right),
$$

where $e_{\pi}$ is the pion charge operator divided by the charge unit $e$, and $r_{\pi}$ is the distance between the center of the quark core and the pion. Our choice of $\hat{Q}=\hat{Q}_{\pi}$ implies that the quark core is spherical and that the entire quadrupole moment comes from the pion p-wave orbital motion. The $\pi^{0}$ terms do not contribute when evaluating the operator $\hat{Q}_{\pi}$ between the wave functions of Eq.(24). We then obtain, e.g., for the spectroscopic $\Delta^{+}$ and $p \rightarrow \Delta^{+}$quadrupole moments

$$
Q_{\Delta^{+}}=-\frac{2}{15}{\beta^{\prime}}^{2} r_{\pi}^{2}, \quad Q_{p \rightarrow \Delta^{+}}=\frac{4}{15} \beta^{\prime} \beta r_{\pi}^{2} .
$$

We have to determine three parameters $\beta, \beta^{\prime}$, and $r_{\pi}$. From the experimental $N \rightarrow \Delta$ quadrupole transition moment, $Q_{p \rightarrow \Delta^{+}}^{e x p} \approx r_{n}^{2}$ [46], we can fix only one of them. Therefore, we also calculate the nucleon and $\Delta$ charge radii in the pion cloud model and obtain

$$
\begin{array}{rlrl}
r_{p}^{2} & =r_{p^{\prime}}^{2}-r_{n}^{2}, & r_{n}^{2}=\beta^{2} \frac{2}{3}\left(r_{p^{\prime}}^{2}-r_{\pi}^{2}\right), \\
r_{\Delta^{+}}^{2}=r_{p^{\prime}}^{2}-r_{\Delta^{0}}^{2}, & r_{\Delta^{0}}^{2}=\beta^{\prime 2} \frac{1}{3}\left(r_{p^{\prime}}^{2}-r_{\pi}^{2}\right) .
\end{array}
$$

Here, $r_{p^{\prime}}^{2}$ is the charge radius of the bare proton. We have assumed that the charge radii of the bare proton and of the bare charged $\Delta$ states are approximately equal and that the bare neutron and $\Delta^{0}$ charge radii are zero. The first equation $r_{p^{\prime}}^{2}=r_{p}^{2}+r_{n}^{2}$ expresses the bare proton charge radius in terms of the experimental isoscalar nucleon charge radius. Subtracting the first and third equations one gets

$$
r_{p}^{2}-r_{\Delta^{+}}^{2}=\left(r_{p^{\prime}}^{2}-r_{\pi}^{2}\right)\left(\frac{1}{3}{\beta^{\prime}}^{2}-\frac{2}{3} \beta^{2}\right)=r_{n}^{2},
$$

where the last equality follows if we choose $\beta^{\prime}=-2 \beta$. When the latter condition is used in Eq.(26), we get

$$
Q_{\Delta^{+}}=Q_{p \rightarrow \Delta^{+}}=r_{n}^{2} .
$$

This is in the same ballpark as the quark model prediction of Eq.(14). From the experimental nucleon charge radii and Eq.(27) one can now determine the remaining parameters $\beta$ and $r_{\pi}$ (see Ref. [33]).
For the spectroscopic quadrupole moment of the proton in the pion cloud model we obtain the following expression

$$
\begin{aligned}
Q^{p} & =\frac{4}{3} \beta^{2} r_{\pi}^{2}\left(\frac{1}{3}\left\langle Y_{0}^{1}\left|P_{2}\right| Y_{0}^{1}\right\rangle+\frac{2}{3}\left\langle Y_{1}^{1}\left|P_{2}\right| Y_{1}^{1}\right\rangle\right) \\
& =\frac{4}{3} \beta^{2} r_{\pi}^{2}\left(\frac{1}{3}\left(\frac{2}{5}\right)+\frac{2}{3}\left(-\frac{1}{5}\right)\right)=0 .
\end{aligned}
$$

The factors $1 / 3$ and $2 / 3$ are the squares of the ClebschGordan coefficients that describe the angular momentum coupling of the bare neutron spin $1 / 2$ with the pion orbital angular momentum $l=1$ to total spin $J=1 / 2$ of the proton. They ensure that the spectroscopic quadrupole moment of the proton is zero. The factors $2 / 5$ and $-1 / 5$ are the expectation values of the Legendre polynomial $P_{2}(\cos \theta)$ evaluated between the pion wave function $Y_{0}^{1}\left(\hat{\mathbf{r}}_{\pi}\right)$ (pion cloud aligned along z-axis) and $Y_{1}^{1}\left(\hat{\mathbf{r}}_{\pi}\right)$ (pion cloud aligned along an axis in the $\mathrm{x}-\mathrm{y}$ plane).

In order to obtain an estimate for the intrinsic quadrupole moment we set by hand each of the coupling coefficients in front of $\left\langle Y_{0}^{1}\left|P_{2}\right| Y_{0}^{1}>\right.$ and $<Y_{1}^{1}\left|P_{2}\right| Y_{1}^{1}>$ equal to $1 / 2$, thereby preserving the sum of coupling coefficients. The cancellation between the two orientations of the cloud then disappears and leads to an overall positive intrinsic quadrupole moment. By this procedure we are undoing the geometric averaging over all angles, which prevents the nonsphericity of the pion cloud from being observed in the laboratory. Furthermore, we note that the first term in Eq.(30), which comes from the $Y_{0}^{1}$ part of the pion wave function, dominates, indicating that the probability for finding the pion in the nucleon is not spherically symmetric but larger at the poles. This term is just the negative of the spectroscopic $\Delta^{+}$quadrupole moment divided by 2 . We take it, properly renormalized, as a measure of the intrinsic quadrupole moment of the nucleon in the pion cloud model. One then finds for the intrinsic quadrupole moment of the proton and the $\Delta^{+}$

$$
Q_{0}^{p}=\frac{8}{15} \beta^{2} r_{\pi}^{2}=-r_{n}^{2}, \quad Q_{0}^{\Delta^{+}}=r_{n}^{2} .
$$

Thus, the intrinsic quadrupole moment of the $p$ is positive and that of the $\Delta^{+}$negative. They are identical in magnitude but opposite in sign. The positive sign of the intrinsic proton quadrupole moment [65] has a simple geometrical interpretation in this model. It arises because the pion is preferably emitted along the spin (z-axis) of the nucleon (see Fig. 4). Thus, the proton assumes a prolate shape. Here, we have neglected the deformation of the bare nucleon (quark core) due to the pressure of the surrounding pion cloud. We emphasize that in this model the deformation comes only from the pion cloud and not from the valence quark core. Previous investigations in a quark model with pion exchange [66] concluded that the nucleon assumes an oblate shape under the pressure of the surrounding pion cloud, which is strongest along the polar axis. However, in these studies the deformed 

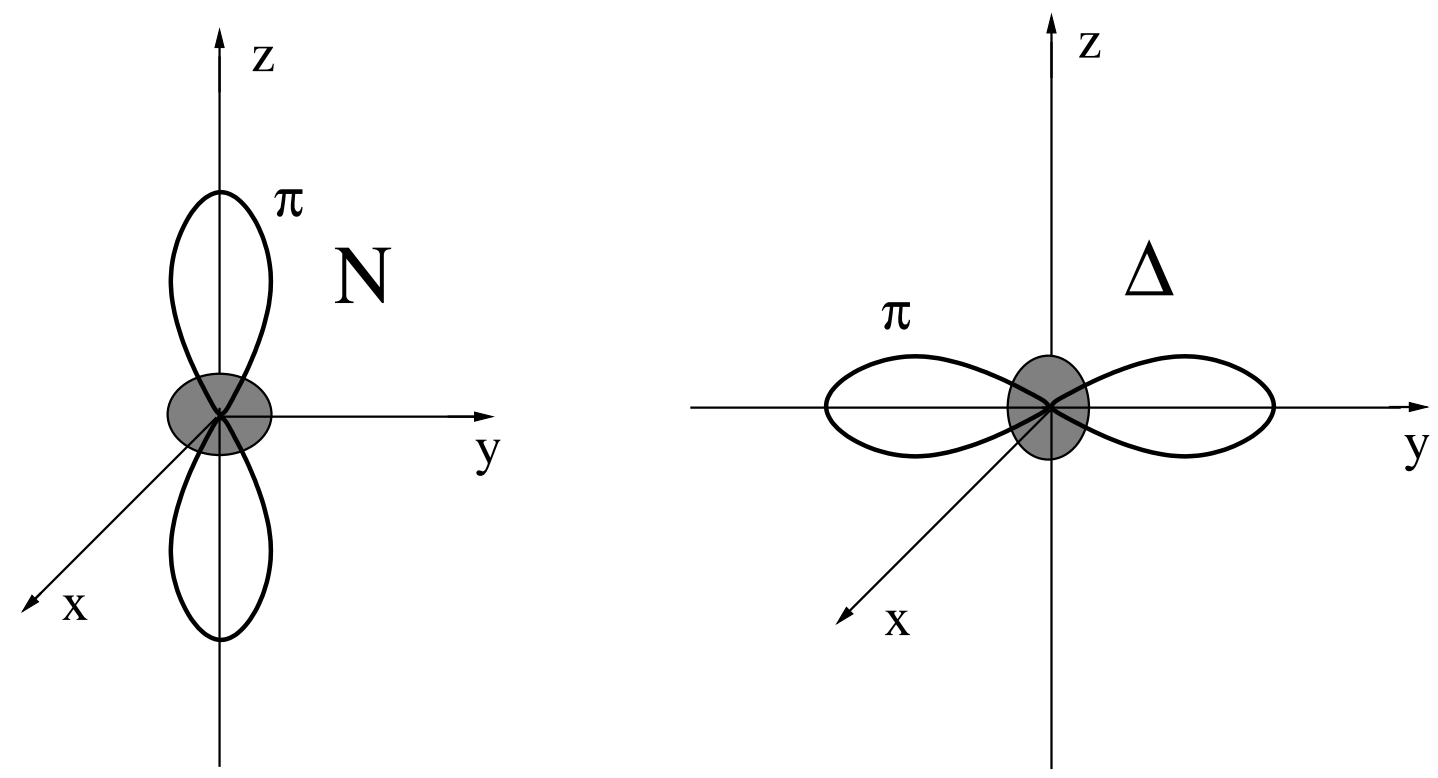

FIG. 4: Intrinsic quadrupole deformation of the nucleon (left) and $\Delta$ (right) in the pion cloud model. In the $N$ the $p$-wave pion cloud is concentrated along the polar (symmetry) axis, with maximum probability of finding the pion at the poles. This leads to a prolate deformation. In the $\Delta$, the pion cloud is concentrated in the equatorial plane producing an oblate intrinsic deformation. Depicted here are the angular ( $p$-wave) parts of the pion wave functions, i.e. $Y_{0}^{1}$ in the case of $N$ and $Y_{1}^{1}$ in the case of $\Delta$ surrounding an almost spherical quark core (from Ref. [33]).

shape of the pion cloud itself was ignored. Inclusion of the latter leads to a prolate deformation that exceeds the small oblate quark bag deformation by a large factor.

\section{ELASTIC FORM FACTORS AND NUCLEON SHAPE}

In this chapter we study the consequences of the relation between $N \rightarrow \Delta$ and elastic $N$ charge form factors for the shape of the nucleon in more detail. To begin with, we generalize the concept of an intrinsic nucleon quadrupole moment to an intrinsic quadrupole form factor of the nucleon. This enables us to draw further conclusions concerning the shape of the nucleon.

\section{A. Intrinsic quadrupole form factor of the nucleon}

The concept of an intrinsic quadrupole moment of the nucleon can be generalized to an intrinsic quadrupole charge distribution and a corresponding quadrupole form factor [34]. To show how this is done, we decompose the proton charge form factor in two terms, a term resulting from a spherically symmetric charge distribution, and a second term due to the intrinsic quadrupole deformation of the physical charge density

$$
\begin{aligned}
& G_{C}^{p}\left(Q^{2}\right)=G_{s y m}^{p}\left(Q^{2}\right)-\frac{1}{6} Q^{2} G_{d e f}\left(Q^{2}\right), \\
& G_{C}^{n}\left(Q^{2}\right)=G_{\text {sym }}^{n}\left(Q^{2}\right)+\frac{1}{6} Q^{2} G_{d e f}\left(Q^{2}\right) .
\end{aligned}
$$

The factor $Q^{2}$ in front of $G_{d e f}$ arises for dimensional reasons and guarantees that the normalization of the charge form factors is preserved. In coordinate space this corresponds to the usual multipole decomposition of the charge density

$$
\rho(\mathbf{r})=\rho_{0}(r) Y_{0}^{0}(\mathbf{r})+\rho_{2}(r) Y_{0}^{2}(\mathbf{r})+\ldots,
$$

where the $\rho_{0}$ part gives rise to $G_{\text {sym }}\left(Q^{2}\right)$ and the $\rho_{2}$ part is connected with $G_{d e f}\left(Q^{2}\right)$.

Concerning the intrinsic charge quadrupole form factor $G_{\text {def }}\left(Q^{2}\right)$, we employ the relation between the $N \rightarrow \Delta$ quadrupole and the elastic neutron charge form factors in Eq.(6), and the relation between the nucleon's intrinsic quadrupole moment and the neutron charge radius in Eq. (22)

$$
G_{d e f}\left(Q^{2}\right)=-\sqrt{2} G_{C 2}^{N \rightarrow \Delta}\left(Q^{2}\right)=\frac{6}{Q^{2}} G_{C}^{n}\left(Q^{2}\right)
$$

with $G_{d e f}(0)=-r_{n}^{2}=Q_{0}^{p}$ as discussed in chapter III. This shows that Eq.(34) is the proper generalization of the intrinsic quadrupole moment to finite momentum transfers. Furthermore, we see that the deviation of the neutron charge form factor from zero and the deviation of the nucleon's charge distribution from spherical symmetry are closely related phenomena. Inserting this expression into Eq.(32) we get

$$
\begin{aligned}
& G_{C}^{p}\left(Q^{2}\right)=G_{s y m}^{p}\left(Q^{2}\right)-G_{C}^{n}\left(Q^{2}\right)=G_{C}^{I S}\left(Q^{2}\right)-G_{C}^{n}\left(Q^{2}\right), \\
& G_{C}^{n}\left(Q^{2}\right)=\frac{1}{6} Q^{2} G_{d e f}\left(Q^{2}\right) .
\end{aligned}
$$




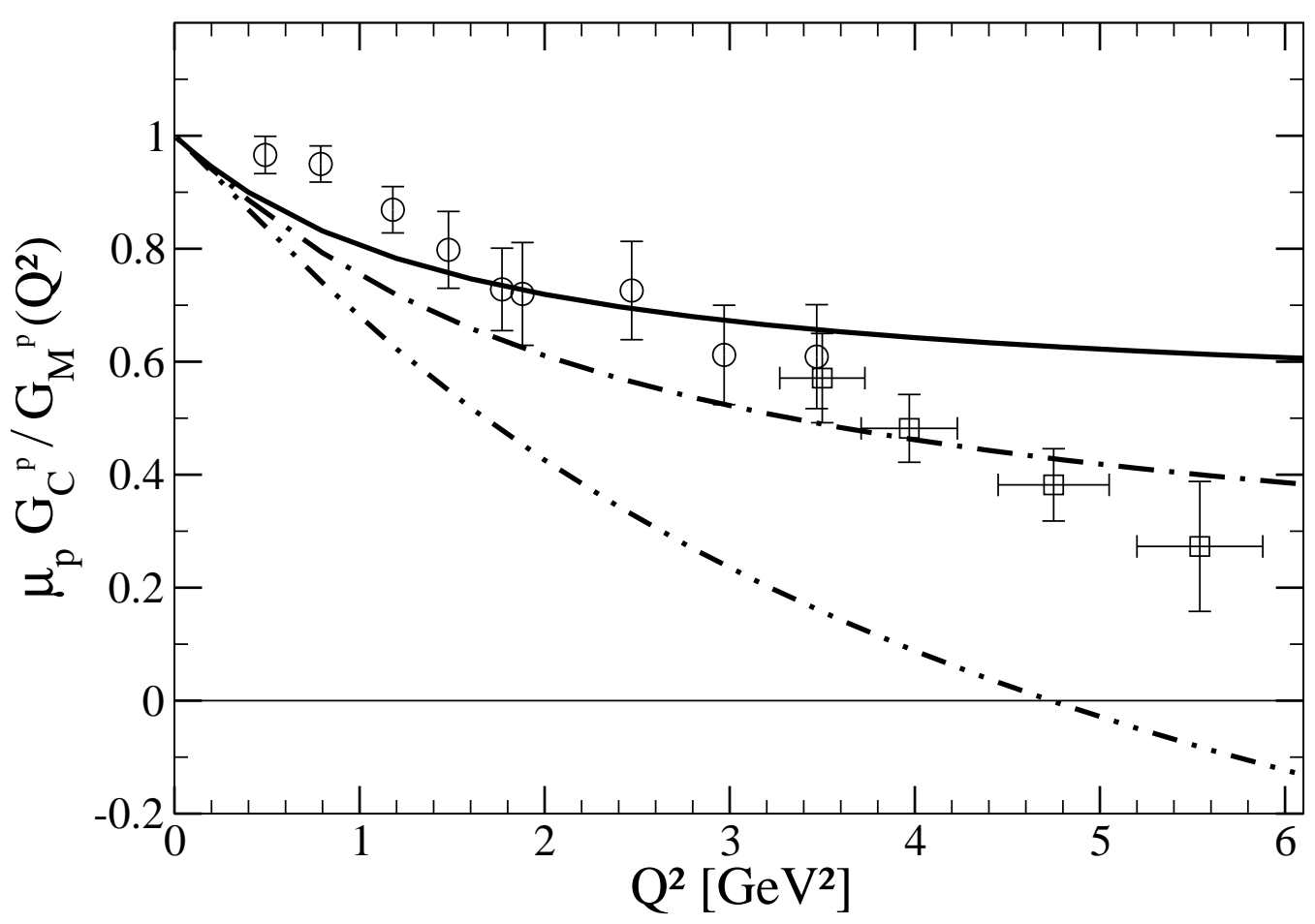

FIG. 5: Proton electromagnetic form factor ratio $R=\mu_{p} G_{C}^{p}\left(Q^{2}\right) / G_{M}^{p}\left(Q^{2}\right)$ as a function of momentum transfer $Q^{2}$ based on the decomposition of $G_{C}^{p}$ in Eq.(35). The three curves correspond to different Galster parameters $d$ for the neutron charge form factor as in Fig. 2.The data are from Ref. [10].

In the case of the neutron, we find that the spherical part is zero, and that the neutron charge form factor is given by the nucleon's intrinsic quadrupole form factor. For the proton, the proposed decomposition implies that the spherically symmetric and the nonspherical intrinsic quadrupole parts are connected respectively with the isoscalar and the neutron charge form factors. Thus, the relation between the $N \rightarrow \Delta$ and neutron charge form factors discussed in chapter II is seen here to have an important implication for the nucleon itself, which can be summarized as: The neutron charge form factor is an observable manifestation and quantitative measure of the intrinsic quadrupole form factor of the nucleon. The latter also manifests itself in the proton charge form factor.

The decomposition suggested in Eq.(35) and its interpretation can also be obtained in the pion cloud model. Generalizing Eq.(27) to finite momentum transfers we obtain a decomposition of the nucleon charge form factors into a bare nucleon and a pion cloud part

$$
\begin{aligned}
& G_{C}^{p}\left(Q^{2}\right)=G_{C}^{p^{\prime}}\left(Q^{2}\right)+G_{C}^{\pi}\left(Q^{2}\right), \\
& G_{C}^{n}\left(Q^{2}\right)=-G_{C}^{\pi}\left(Q^{2}\right),
\end{aligned}
$$

where the bare neutron contribution to the neutron charge form factor is zero. Comparison of these expressions with Eq.(35) shows that the bare proton contribution can be identified with the spherically symmetric part while the pion contribution is associated with the quadrupole deformation part as follows

$$
\begin{aligned}
& G_{C}^{p^{\prime}}\left(Q^{2}\right)=G_{C}^{I S}\left(Q^{2}\right)=G_{s y m}^{p}\left(Q^{2}\right), \\
& G_{C}^{\pi}\left(Q^{2}\right)=-G_{C}^{n}\left(Q^{2}\right)=-\frac{1}{6} Q^{2} G_{d e f}\left(Q^{2}\right) .
\end{aligned}
$$

In summary, both the quark model and the pion cloud model suggest a decomposition of the nucleon charge form factors into a spherically symmetric quark core or bare nucleon part, and an intrinsic quadrupole part arising from quark-antiquark pairs or pion degrees of freedom. Furthermore, both models show that the neutron charge form factor is an observable manifestation of the intrinsic quadrupole charge form factor of the nucleon.

\section{B. Charge radii and nucleon shape}

According to the proposed decomposition of the nucleon charge form factors in Eq.(32), the nucleon charge radii can be written as a sum of two terms, a spherically symmetric and a nonspherical intrinsic quadrupole contribution. The latter deformation contribution to the proton charge radius which makes the charge radius bigger is given by the negative neutron charge radius

$$
\begin{aligned}
& r_{p}^{2}=r_{p, \text { sym }}^{2}+r_{d e f}^{2}=r_{p}^{2}+r_{n}^{2}-r_{n}^{2} \\
& r_{n}^{2}=r_{n, \text { sym }}^{2}-r_{d e f}^{2}=-r_{d e f}^{2} .
\end{aligned}
$$


If written in this way, we see that the spherical contribution to the proton charge radius is given by the isoscalar charge radius and the deformation contribution by the negative of the neutron charge radius. In the case of the neutron, the spherical part is zero and all of the neutron charge radius is due to the intrinsic deformation of its charge distribution. Eqs.(38) are the quark model counterparts of the pion cloud model formulae in Eq.(27).

\section{Nucleon electromagnetic form factor ratio}

Nucleon recoil polarization measurements in elastic electron-proton scattering render it possible to extract the charge and magnetic form factors of the nucleon with higher precision than with the Rosenbluth separation method that has mainly been used in the past. An important result of these experiments is that the proton charge over magnetic form factor ratio shows an almost linear decrease with increasing momentum transfer $[10,13]$. In the following we show that this result can be understood with the help of the decomposition of the charge form factor into two terms as discussed in the previous sections.

Using Eq.(35) for $G_{C}^{p}$ we obtain for the proton form factor ratio the following expression

$$
\begin{aligned}
R\left(Q^{2}\right) & =\mu_{p} \frac{G_{C}^{p}\left(Q^{2}\right)}{G_{M}^{p}\left(Q^{2}\right)} \\
& =\frac{\mu_{p}}{G_{M}^{p}\left(Q^{2}\right)}\left(G_{C}^{I S}\left(Q^{2}\right)-G_{C}^{n}\left(Q^{2}\right)\right) .
\end{aligned}
$$

If we insert experimental results for the isoscalar and neutron charge form factors, the measured ratio $R$ is reproduced. Thus, our splitting of $G_{C}^{p}$ in two terms does not modify the measured ratio $R$ in any way. The advantage of the proposed decomposition is that it provides a physical interpretation of the momentum dependence of $R\left(Q^{2}\right)$. From the second equality in Eq.(39) we observe that the decrease of $R$ with increasing $Q^{2}$ comes from the intrinsic quadrupole form factor of the nucleon, which is closely related to the neutron charge form factor via Eq.(34). This is qualitatively shown in Fig. 5, where we have used a simple dipole for the isoscalar charge and proton magnetic form factors, and a Galster parametrization for the neutron charge form factor. As mentioned above, an exact agreement between the present theory and experiment could be established by inserting experimental data for $G_{C}^{I S}$ and $G_{C}^{n}$ instead of the above parametrizations. Nevertheless, even the qualitative parametrizations employed here show that the observed decrease of $R$ can be explained by a nonspherical part in the proton charge distribution that is described by the neutron charge form factor. In coordinate space the latter leads to a more spread out charge distribution compared to the magnetic dipole distribution [3, 34].

As clearly seen from Eq.(35) the same quark-antiquark degrees of freedom, which give rise to the nonzeroness of
$G_{C}^{n}\left(Q^{2}\right)$ and the related nonsphericity of the neutron's charge distribution also show up in the proton charge form factor. This is not only true at very low $Q^{2}$ (charge radii) and very high momentum transfers (decrease of $R$ ) but also at intermediate $Q^{2}$. Our results provide an independent confirmation of the observation made in Ref. [4] that the dip structure observed in the proton charge form factor at around $Q^{2}=0.4 \mathrm{GeV}^{2}[4]$ is due to exactly the same structure in the neutron charge form factor. Furthermore, according to the present theory a similar structure should be observed in the $N \rightarrow \Delta$ charge quadrupole form factor.

\section{SUMMARY}

Advances in electron-nucleon scattering experiments have revealed more detailed information on the nucleon charge form factors at high momentum transfers, and have provided clear evidence for a small but nonvanishing charge quadrupole transition form factor in the excitation of the lowest-lying nucleon resonance $\Delta(1232)$.

Using a quark model incorporating spin-flavor symmetry and its breaking by spin-dependent two- and threequark charge operators, we have derived a relation between the neutron charge form factor and the $N \rightarrow \Delta$ charge quadrupole form factor. With the help of this relation we have expressed the $C 2 / M 1\left(Q^{2}\right)$ ratio for $\Delta$ electroexcitation in terms of the elastic neutron form factor ratio $G_{C}^{n} / G_{M}^{n}\left(Q^{2}\right)$. Comparison with the data has shown that the two data sets satisfy the proposed relation within the experimental accuracy over a wide range of momentum transfers.

To draw a first conclusion concerning the geometric shape of the nucleon from these experiments we have pointed out that information on the shape of a spin $1 / 2$ system can be obtained from its intrinsic quadrupole moment. Employing a quark model with exchange currents and a pion cloud model, we have shown that the intrinsic nucleon quadrupole moment is closely related to the measured $N \rightarrow \Delta$ quadrupole moment. In particular, we have found that the nucleon's intrinsic quadrupole moment is positive and can be expressed by the modulus of the neutron charge radius. Thus, the nucleon ground state charge distribution posseses a prolate shape.

To obtain further information on the nucleon's charge distribution, we have decomposed the nucleon charge form factors in a spherically symmetric and an intrinsic quadrupole part. For the latter we have employed the relation between the $N \rightarrow \Delta$ quadrupole and neutron charge form factors. This decomposition shows that the neutron charge form factor is an observable manifestation of the intrinsic quadrupole deformation of the nucleon. In the case of the proton, the intrinsic quadrupole form factor also leads to observable consequences. In particular, at high $Q^{2}$ it provides an explanation of the experimentally observed decrease of the proton charge over magnetic form factor ratio. 
As to the physical origin of nucleon deformation, all available information suggests that collective quarkantiquark degrees of freedom, which in the quark model are effectively described by two- and three-quark charge operators, and in the pion cloud model by explicit pion degrees of freedom, are responsible for the nonzero neutron charge and $N \rightarrow \Delta$ quadrupole transition form factors, and thus for a nonzero intrinsic quadrupole form factor of the nucleon.

Concerning the nucleon's magnetic form factors, we can decompose them into leading dipole and an intrinsic octupole part, which is related to the observable magnetic octupole form factor of the $\Delta$ resonance. Because the latter is only nonvanishing if we include third order $\mathrm{SU}(6)$ symmetry breaking or three-quark currents, its effect on the magnetic form factors of the nucleon is expected to be much smaller than in the case of the charge form factors. We hope to discuss these matters in a forthcoming publication.

In summary, we have provided some arguments that the relation between the inelastic $N \rightarrow \Delta$ quadrupole and elastic neutron charge form factors is based on the underlying SU(6) spin-flavor symmetry of QCD, which unites the $N$ and $\Delta$ into a common $\mathbf{5 6}$ dimensional ground state supermultiplet. In the exact symmetry limit, $N$ and $\Delta$ masses are degenerate. In addition, the neutron charge form factor as well as the $\Delta$ and $N \rightarrow \Delta$ quadrupole form factors are exactly zero. The breaking of this symmetry by spin-flavor dependent two- and three-quark operators in the Hamiltonian and charge operator leads to the lifting of the mass degeneracy, a nonzero charge form factor of the neutron, as well as nonzero $\Delta$ and $N \rightarrow \Delta$ transition quadrupole form factors, in agreement with experiment. Moreover, the broken symmmetry predicts that the $N \rightarrow \Delta$ quadrupole transition form factor is related to the ground state neutron charge form factor. This seems to be quite well satisfied in nature. Finally, this relation has interesting implications for the elastic charge form factors of the nucleon. It suggests that the decrease of the proton charge form factor at high $Q^{2}$ beyond the simple dipole behavior is due to an intrinsic quadrupole deformation of the proton's charge density. In the case of the neutron, it leads to the conclusion that the neutron charge form factor is a direct measure of the nucleon's intrinsic quadrupole deformation.

\section{Note added in December 2007}

Recently, an analysis of the world pion electroproduction cross section data [67] has led to an empirical $C 2 / M 1$ ratio that is in agreement with our prediction in Eq.(9) also at high momentum transfers.

\section{Acknowledgments}

I would like to thank Aron Bernstein and Costas Papanicolas for their interest in this work.
[1] E. E. Chambers and R. Hofstadter, Phys. Rev. 103, 1454 (1956).

[2] G. G. Simon, F. Borkowski, Ch. Schmitt and V. H. Walther, Z. Naturf. 35a, 1 (1980).

[3] J.J. Kelly, Phys. Rev. C 66, 065203 (2002).

[4] J. Friedrich and Th. Walcher, Eur. Phys. J. A 17 607, (2003); hep-ph/0303054.

[5] F. Halzen and A. D. Martin, Quarks and Leptons, Wiley, New York, 1984.

[6] Here, I use $G_{C}^{N}\left(Q^{2}\right)$ for the nucleon charge (Coulomb) form factors instead of the usual notation $G_{E}^{N}\left(Q^{2}\right)$ in order to emphasize the parallels between elastic and inelastic charge form factors.

[7] The four momentum is defined as $Q^{2}=:-q_{\mu} q^{\mu}=\omega^{2}-$ $\mathbf{q}^{2}$, with $q^{\mu}=(\omega, \mathbf{q})$ where $\omega=\epsilon_{i}-\epsilon_{f}$ is the energy transfer and $\mathbf{q}=\mathbf{k}_{i}-\mathbf{k}_{f}$ the three-momentum transfer of the virtual photon. Here, $\left(\epsilon_{i}, \mathbf{k}_{i}\right)$ and $\left(\epsilon_{f}, \mathbf{k}_{f}\right)$ are the electron energy and three-momentum in the initial and final states.

[8] Experimentally, the cross section is measured for different incident energies $\epsilon_{i}$ and scattering angles $\Theta$ while keeping $Q^{2}$ and $\omega$ fixed. The ratio $r=(d \sigma / d \Omega)_{e l} /\left(\sigma_{M} f_{r e c}\right)$ is then only a function of $\tan ^{2}(\Theta / 2)$. When plotting the measured ratio $r$ against $\tan ^{2}(\Theta / 2)$ the resulting curve is a linear function with a slope given by $G_{M}^{N^{2}}$ and an intercept by $\left(G_{C}^{N^{2}}+\tau G_{M}^{N}\right) /(1+\tau)$. The extraction of the charge form factor becomes increasingly more inaccurate at higher momentum transfers because of its suppression by the factor $\tau$.

[9] G. Dillon and G. Morpurgo, Phys. Lett. B459, 321 (1999).

[10] M. K. Jones, Phys. Rev. Lett. 84, 1398 (2000); O. Gayou, Phys. Rev. Lett. 88, 092301 (2002).

[11] The different results for the nucleon form factors obtained from both types of experiment can to some extent be understood in terms of two-photon exchange contributions, which affect the extraction of the form factors from the unpolarized cross section whereas the recoil polarization measurements are less sensitive to these corrections [12].

[12] P. A. M. Guichon and M. Vanderhaeghen, Phys. Rev. Lett. 91, 142303 (2003).

[13] J. Arrington, Phys. Rev. C 68, 034325 (2003).

[14] H.F. Jones and M.D. Scadron, Ann. Phys. 81, 1 (1973); R.C.E. Devenish, T.S. Eisenshitz, and J. Körner, Phys. Rev. D 14, 3063 (1976); M.M. Giannini, Rep. Progr. Phys. 54 (1990) 453; M. Warns, H. Schröder, W. Pfeil, and H. Rollnik, Z. Phys. C 45, 627 (1990).

[15] C. E. Carlson, Phys. Rev. D34, 2704 (1986).

[16] T. DeForest, Jr. and J. D. Walecka, Adv. Phys. 15, 1 (1966).

[17] A.M. Bernstein, Eur. Phys. J. A 17, 349 (2003); C. Mertz et al., Phys. Rev. Lett. 86, 2963 (2001); C.N. Papanicolas, Eur. Phys. J. A 18, 141 (2003); C.N. Papanicolas, Workshop on Electron Nucleus Scattering, Marciana Marina, Italy, 1988, World Scientific, 1989, pg. 41-61. 
[18] A.J. Buchmann, E. Hernández, A. Faessler, Phys. Rev. C 55, 448 (1997).

[19] L.D. van Buuren et al., Phys. Rev. Lett. 89, 012001 (2002).

[20] K. Joo et al., Phys. Rev. Lett. 88, 122001 (2002).

[21] P. Bartsch et al., Phys. Rev. Lett. 88, 142001 (2002); Th. Pospischil et al., Phys. Rev. Lett. 86, 2959 (2001).

[22] S. Stave et al., Eur. Phys. J. A 30, 471 (2006); nuclex/0604013

[23] D. Elsner et al., Eur. Phys. J. A 27, 91 (2006).

[24] N. F. Sparveris et al., Phys. Rev. Lett. 94, 022003 (2005).

[25] M. Ungaro et al., Phys. Rev. Lett. 97, 112003 (2006).

[26] A. Idilbi, X. Ji, J.P. Ma, Phys. Rev. D 69, 014006 (2004).

[27] L. Tiator, D. Drechsel, S.S. Kamalov, and S.N. Yang, Eur. Phys. J. A 17, 357 (2003).

[28] E. Jenkins, X. Ji, and A.V. Manohar, Phys. Rev. Lett. 89, 242001 (2002).

[29] C. Alexandrou, Nucl. Phys. Proc. Suppl. 128, 1 (2004), arXiv:nucl-th/0311007.

[30] A.J. Buchmann, J.A. Hester, and R.F. Lebed, Phys. Rev. D 66, 056002 (2002).

[31] F. Gross and P. Agbakpe, Phys. Rev. C 73, 015203 (2006).

[32] V. Pascalutsa, M. Vanderhaeghen, S.N. Yang, Phys. Rep. 437, 125 (2007); hep-ph/0609004

[33] A.J. Buchmann and E.M. Henley, Phys. Rev. C 63, 015202 (2001); Phys. Rev. D 65, 073017 (2002).

[34] A. J. Buchmann, Can. J. Phys. 83,455 (2005).

[35] M. Gell-Mann and Y. Ne'eman, The Eightfold Way, W. A. Benjamin, New York 1964.

[36] F. Gürsey and L.A. Radicati, Phys. Rev. Lett. 13, 173 (1964).

[37] B. Sakita, Phys. Rev. Lett. 13, 643 (1964).

[38] M.A.B. Beg, B.W. Lee, and A. Pais, Phys. Rev. Lett. 13, 514 (1964).

[39] J.-L. Gervais and B. Sakita, Phys. Rev. D 30, 1795, (1984).

[40] R.F. Dashen, E. Jenkins, and A.V. Manohar, Phys. Rev. D 51, 3697 (1995).

[41] R.F. Lebed, Czech. J. Phys. 49, 1273 (1999); nuclth/9810080. This paper contains a pedagogical introduction to this method.

[42] R.F. Lebed, Phys. Rev. D 51, 5039 (1995).

[43] A.J. Buchmann, Nucl. Phys. A 670, 174c (2000); hepph/0208045; hep-ph/0207368; hep-ph/0301031.

[44] G. Dillon and G. Morpurgo, hep-ph/0011202

[45] R.F. Lebed and D.R. Martin, Phys.Rev. D 70, 016008 (2004).

[46] G. Blanpied et al., Phys. Rev. Lett. 79 , 4337 (1997); Phys. Rev. C 64025203 (2001).

[47] P. Grabmayr and A.J. Buchmann, Phys. Rev. Lett. 86, 2237 (2001). The relation between the Galster parameters and the neutron charge form factor moments are: $a=2 M_{N}^{2} r_{n}^{2} /\left(3 \mu_{n}\right)$ and $d=2 M_{N}^{2}\left[r_{n}^{4} /\left(10 r_{n}^{2}\right)-4 / \Lambda^{2}\right]$.
[48] A.J. Buchmann, Phys. Rev. Lett. 93, 212301 (2004).

[49] Our definition is equivalent to the definition $C 2 / M 1=$ : $-|\mathbf{q}| /\left(2 M_{\Delta}\right)\left(G_{C}^{*} / G_{M}^{*}\right)$ based on the dimensionless transition form factors $G_{C}^{*}$ and $G_{M}^{*}$ employed by a number of authors [14]. The advantage of our $G_{C 2}^{N \rightarrow \Delta}\left(Q^{2}\right)$ and $G_{M 1}^{N \rightarrow \Delta}\left(Q^{2}\right)$ is that they correspond to the familiar electromagnetic multipole moments in the $Q \rightarrow 0$ limit.

[50] In this frame $|\mathbf{q}|=\left(Q^{2}+\left(M_{\Delta}^{2}-M_{N}^{2}-Q^{2}\right)^{2} /\left(4 M_{\Delta}^{2}\right)\right)^{1 / 2}$, where $M_{\Delta}=1232 \mathrm{MeV}$ is the $\Delta$ mass. For $Q^{2}=0$ one obtains $|\mathbf{q}|=\left(M_{\Delta}^{2}-M_{N}^{2}\right) /\left(2 M_{\Delta}\right)=258 \mathrm{MeV}$.

[51] S. Galster et al., Nucl. Phys. B 32, 221 (1971).

[52] R. Beck et al., Phys. Rev. Lett. 78, 606 (1997).

[53] R. Gothe, Proceedings of NSTAR 2000, Newport News, World Scientific, 2001, eds. V. Burkert et al., pg. 31.

[54] R. Siddle et al., Nucl. Phys. B 35, 93 (1971)

[55] V.V. Frolov et al., Phys. Rev. Lett. 82, 45 (1998). In this paper the $C 2 / M 1$ ratio is extracted from the cross section using two methods: (i) a multipole fit to the pion angular distributions, (ii) a Lagrangian model. I take the results of method (i), which uses fewer assumptions. In this context see also [57].

[56] J.C. Alder et al., Nucl. Phys. B 46, 573 (1972).

[57] J. J. Kelly, Phys. Rev. C 72, 048201 (2005); Phys. Rev. C 72, 059901(E) (2005).

[58] The real photon data for $E 2 / M 1(0)[46,52]$ can be used to estimate $C 2 / M 1(0)$, because according to Siegert's theorem the electric quadrupole form factor $G_{E 2}^{N \rightarrow \Delta}\left(Q^{2}\right)$ and the charge quadrupole form factor $G_{C 2}^{N \rightarrow \Delta}\left(Q^{2}\right)$ are related in the limit $|\mathbf{q}| \rightarrow 0$. Even at $|\mathbf{q}|=258 \mathrm{MeV}$, i.e. at $Q^{2}=0$, Siegert's theorem is very well satisfied [59].

[59] A.J. Buchmann, E. Hernández, U. Meyer, A. Faessler, Phys. Rev. C 58, 2478 (1998).

[60] A.J. Buchmann, E. Hernández, and K. Yazaki, Phys. Lett. B269, 35 (1991); Nucl. Phys. A 569, 661 (1994).

[61] A. J. Buchmann and R. F. Lebed, Phys. Rev. D 62, 096005 (2000). As remarked on in the paragraph before Eq.(3.2) the same formalism will lead to a relation between $N$ and $\Delta$ masses $M_{p}-M_{\Delta^{+}}=M_{n}-M_{\Delta^{0}}$ analogous to the charge radius relation $r_{p}^{2}-r_{\Delta^{+}}^{2}=r_{n}^{2}-r_{\Delta^{0}}^{2}$. The mass relation is experimentally well satisfied.

[62] M. A. Beg and V. Singh, Phys. Rev. Lett. 13, 418 (1964).

[63] A. Bohr and B. Mottelson, Nuclear Structure II, Benjamin, Reading, MA, 1975.

[64] E. M. Henley and W. Thirring, Elementary Quantum Field Theory, McGraw-Hill, New York, 1962.

[65] After dividing by the negative sign of the $\pi^{-}$cloud, the neutron's intrinsic quadrupole moment is also positive, i.e., $Q_{0}^{n}=Q_{0}^{p}$.

[66] G. E. Brown, Nucl. Phys. A 358, 39c (1981); V. Vento, G. Baym, and A. D. Jackson, Phys. Lett. 102B, 97 (1981).

[67] D. Drechsel, S.S. Kamalov, L. Tiator, Eur. Phys. J. A 34, 69 (2007). 